\title{
A cycle of success: the interdependence of surgery and science
}

\author{
M. Schneider • T. Welsch • M. Kremer • M. W. Büchler
}

(C) Springer-Verlag 2012

Securing progress in clinical surgery and delineating novel treatment options to meet emerging clinical challenges remains a formidable task of an academic department of surgery. This endeavor requires not only a strong commitment to research by the surgeon. Equally important are appropriate research structures and a sense of fruitful collaboration between surgeons and basic scientists from the various fields of biomedical research. Undoubtedly, a close exchange between clinicians and scientists is paramount in order to transform unresolved clinical problems into appropriate scientific questions, which can be further tackled at the laboratory bench (Fig. 1). Conversely, a frequent dialogue between the scientist and the surgeon is necessary in order to pinpoint the clinically relevant aspects of basic laboratory research. Exploiting the latter to implement novel surgical treatment options is the aim of translational research - which represents an important cornerstone of surgical science. Last but not least, the need for frequent evaluation of novel and already existent therapeutic principles strongly necessitates clinical research, encompassing multicenter trials, metaanalyses and systematic reviews [1].

The interplay between surgery and basic science might thus be illustrated as a cycle involving both the clinician and the scientist (Fig. 1). The tighter and more proficient the relationship between its contributors, the more efficiently will this cycle lead towards progress in clinical surgery. But

\footnotetext{
M. Schneider · T. Welsch • M. Kremer • M. W. Büchler $(\bowtie)$ Department of Surgery, University of Heidelberg, Im Neuenheimer Feld 110, 69120, Heidelberg, Germany e-mail: Markus.Buechler@med.uni-heidelberg.de
}

how can we ensure a fruitful dialogue between surgery and science? Several structural concepts are feasible; probably the most evident of them is a close collaboration between surgical departments and independent surgical research facilities located at the same site-or surgical research units integrated into clinical departments. A step further in this direction is the integration of research-minded surgeons into interdisciplinary research consortia comprising clinicians and scientists from various research fields, who join forces to advance patient-oriented experimental and clinical research. Independent funding organisations do increasingly acknowledge the unprecedented value of such research consortia and support the implementation of interdisciplinary research programmes combining the areas of clinical, applied and basic research to explore topical research questions. It will become increasingly important for surgeons to pick these opportunities. Last but not least, research structures integrating basic scientists and clinical surgeons are quintessential to promote the careers of research-minded surgeons [2].

This issue of Langenbeck's Archives of Surgery comprises a series of scientific review articles, contributed by German surgeon-scientists on occasion of the 129th annual conference of the German Society of Surgery. These articles altogether reflect current and fruitful attempts at advancing insights into basic molecular and cellular processes that are immediately relevant for clinical practice. How, for instance, might genetic and molecular characteristics of cancer cells be exploited for the prevention of pancreatic cancer [3], to bypass the resistance of colorectal tumours against chemoand radiation therapy [4], or to develop suitable biomarkers for oncologic treatment [5]? What are immune mechanisms 


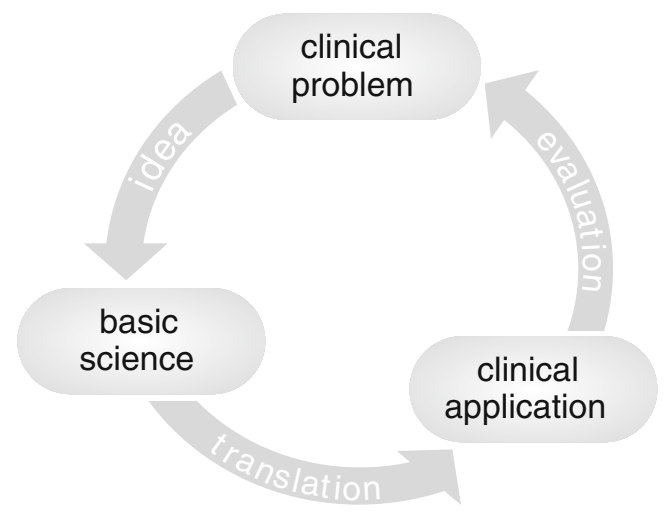

Fig. 1 Interplay between basic science, translational research and clinical studies in clinical problem solving

underlying postoperative intestinal dysmotility - and how can they be influenced to devise innovative strategies for the treatment of postoperative ileus [6]? How do alterations in hepatic blood flow impact on liver regeneration and, thus, on clinical outcomes following surgical liver resection [7]?

Collectively, these contributions, along with the science abstracts presented at the current annual meeting of the German Society of Surgery (which are likewise included in this issue), vividly exemplify a successful partnership between science and surgery and encourage its continued endorsement in order to advance the field of surgery.

\section{References}

1. Knebel P, Kühn S, Ulrich A, Büchler MW, Diener M (2012) The Study Centre of the German Surgical Society: current trials and results. Langenbeck's Arch Surg 397:611-618

2. Menger M, Schilling $M$, Schäfers $H$, Pohlemann T, Laschke $M$ (2012) How to ensure the survival of the surgeon-scientist? The Homburg program. Langenbeck's Arch Surg 397:619-622

3. Fendrich V (2012) Chemoprevention of pancreatic cancer-one step closer. Langenbeck's Arch Surg 396:495-505

4. Grade M, Wolff H, Gaedcke J, Ghadimi M (2012) The molecular basis of chemoradiosensitivity in rectal cancer: implications for personalized therapies. Langenbeck's Arch Surg 397:543-555

5. Steinert G, Schölch S, Koch M, Weitz J (2012) Biology and significance of circulating and disseminated tumor cells in colorectal cancer. Langenbeck's Arch Surg 397:535-542

6. Wehner S, Vilz T, Stoffels B, Kalff J (2012) Immune mediators of postoperative ileus. Langenbeck's Arch Surg 397:591-601

7. Abshagen K, Eipel C, Vollmar B (2012) A critical appraisal of the hemodynamic signal driving liver regeneration. Langenbeck's Arch Surg 396:579-590 